Provided for non-commercial research and education use. Not for reproduction, distribution or commercial use.

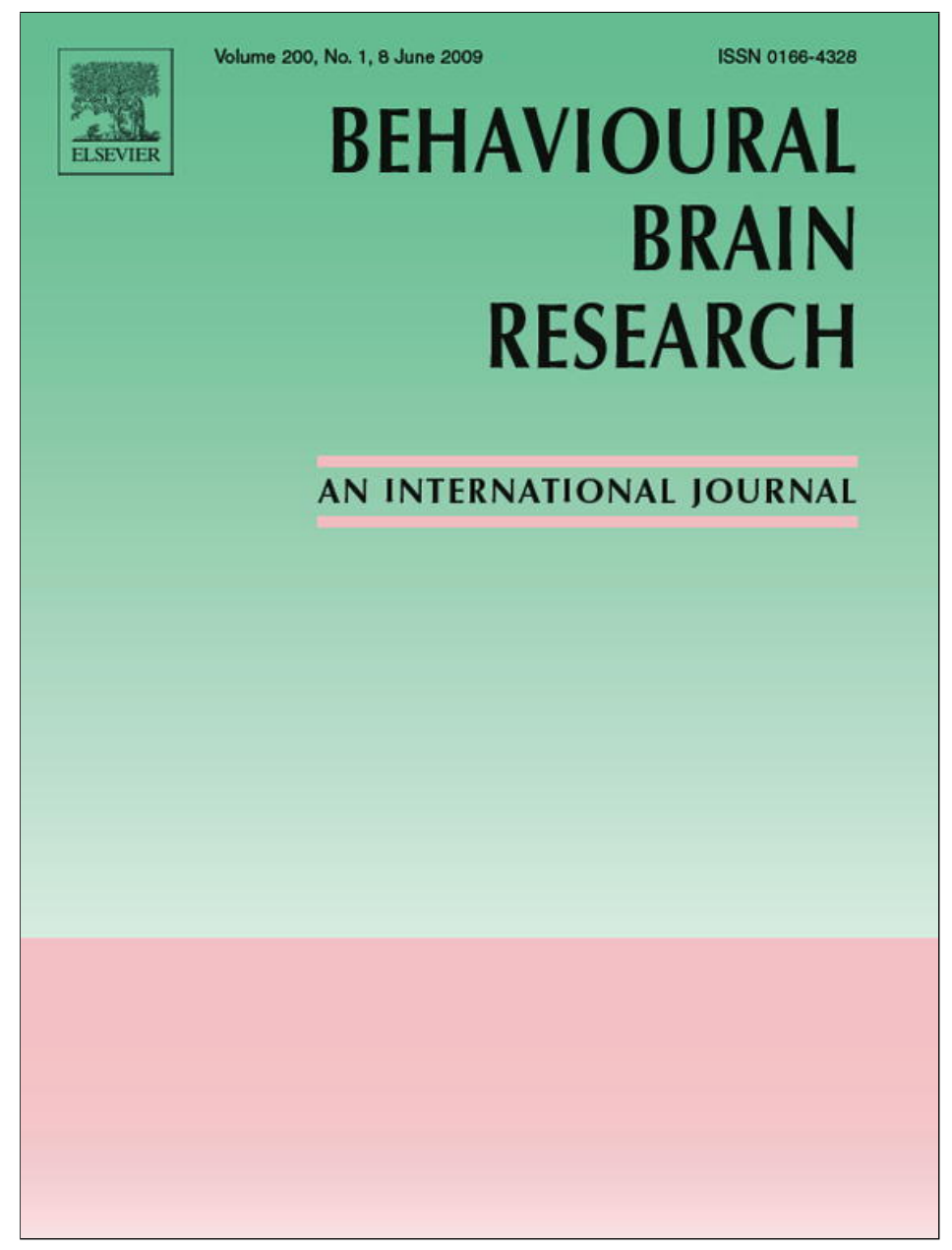

This article appeared in a journal published by Elsevier. The attached copy is furnished to the author for internal non-commercial research and education use, including for instruction at the authors institution and sharing with colleagues.

Other uses, including reproduction and distribution, or selling or licensing copies, or posting to personal, institutional or third party websites are prohibited.

In most cases authors are permitted to post their version of the article (e.g. in Word or Tex form) to their personal website or institutional repository. Authors requiring further information regarding Elsevier's archiving and manuscript policies are encouraged to visit:

http://www.elsevier.com/copyright 
Research report

\title{
Differential place and response learning in horses displaying an oral stereotypy
}

\author{
Matthew Parker ${ }^{\mathrm{a}, *}$, Sebastian D. McBride ${ }^{\mathrm{b}}$, Edward S. Redhead ${ }^{\mathrm{a}}$, Deborah Goodwin ${ }^{\mathrm{a}}$ \\ a School of Psychology, University of Southampton, Highfield, Southampton, Hampshire SO17 1BJ, UK \\ ${ }^{\mathrm{b}}$ Royal Agricultural College, Cirencester, Gloucestershire GL7 6JS, UK
}

\section{A R T I C L E I N F O}

\section{Article history:}

Received 2 October 2008

Received in revised form

23 December 2008

Accepted 31 December 2008

Available online 8 January 2009

\section{Keywords:}

Horse

Stereotypy

Habit

Place/response

\begin{abstract}
A B S T R A C T
Significant similarities exist between the neural and behavioural features of environmentally and drug-induced stereotypy. For example, exposure to dopamine agonists, such as amphetamine, induces stereotypy and causes alterations in midbrain neurophysiology similar to those observed following chronic stress. An additional behavioural feature of these neural changes in the drug-induced phenotype is an enhanced rate of switching from response-outcome (R-O) to stimulus-response (S-R) learning. The aim of the current experiment was to examine R-O and S-R learning in horses displaying environmentally induced oral stereotypies. This was achieved by employing variations of the place/response paradigm. In Experiment 1, we found that crib-biting horses displayed 'response' learning after 20 learning trials, whereas non-crib-biting controls tended to display 'place' learning throughout the experiment. In Experiment 2, we used a modified version of the procedure, in which the subjects were introduced to the maze from different start points and forced always to turn the same way. We found that the crib-biters acquired the task at a faster rate suggesting again that this group was displaying 'response' learning. Finally, in Experiment 3, we carried out an arena test to ensure that crib-biters were capable of 'place' learning. These results are the first to show that horses displaying an oral stereotypy, a behavioural phenotype previously associated with stress-induced perturbations of the basal ganglia, preferentially use 'response' learning. The findings are discussed in relation to the search for an aetiological model of stereotypy.
\end{abstract}

() 2009 Elsevier B.V. All rights reserved.

\section{Introduction}

Stereotypic behaviours are characterised as repetitive, rigid, idiosyncratic and topographically invariant response patterns that can either be environmentally [16] or pharmacologically [7] induced. Environmentally induced stereotypies are typically seen in laboratory, domestic or non-feral species maintained in suboptimal environmental conditions, and they are often cited as an indicator of inadequate welfare provision [16]. In horses, a variety of stereotypies have been identified (see [12] for a comprehensive review), the most common of which is crib-biting [18]. Crib-biting can be described as the horse grasping a solid object between the upper and lower incisors, inhaling air into the oesophagus and emitting an audible 'grunt' [19].

Chronic stress during early development of an inbred strain of mice (DBA) results in a variety of behavioural and neurobiological changes, including sensitisation to the locomotor effects of dopamine agonists, increased behavioural stereotypy

\footnotetext{
* Corresponding author. Present address: Department of Veterinary Clinical Sciences, Royal Veterinary College, Hawkshead Lane, North Mymms, Hatfield, Hertfordshire AL9 7TA, UK. Tel.: +44 1707667095.

E-mail address: mparker@rvc.ac.uk (M. Parker).
}

and upregulation of dopamine transmission in the mesoaccumbens pathway (ventral tegmental area to nucleus accumbens) [1]. These behavioural and physiological changes were not observed in another strain of inbred mice (C57) suggesting that stress-induced behavioural sensitisation and stereotypy development is a result of a genotype $\times$ stress interaction [1]. Similar changes in dopamine physiology were observed in crib-biting compared to control horses [17] with the former having increased dopamine D1 and D2 receptors in the ventral striatum as well as decreased density of D1 receptor subtypes in the caudate. This suggests that alteration of basal ganglia physiology is common to environmentally induced stereotypy development in a range of species.

Given the primary functionality of the basal ganglia (i.e., learning, motivation, reward and action selection [8,28-32]), it would be expected that animals displaying environmentally induced stereotypies would also exhibit other behavioural or learning differences. Within standard extinction paradigms and sequential responding in a gambling task, stereotypy in songbirds was positively correlated with latency to extinguish conditioned responses in the extinction procedures and increased 'predictable' responding during the gambling task [9]. The extinction findings have recently been extended to include non-laboratory species (e.g. horses [11] and bears [27]). Further learning differences were exhibited by crib-biting horses in an instrumental choice procedure, with this 


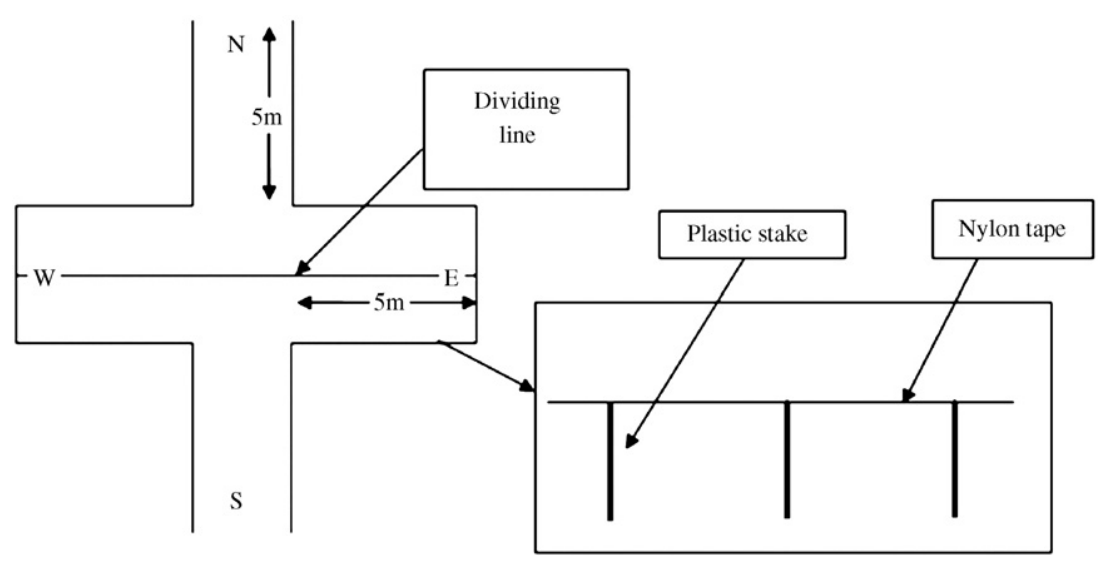

Fig. 1. Illustration of the cross-maze (Experiments 1 and 2). In Experiment 1, during training trials, the horse was led into the maze in the south arm, and the food bucket was located in the east arm, with the west arm containing an identical empty bucket. During the probe trial, the horse was led in through the north arm, with both the east and west arms containing empty buckets. The dividing line in the centre was put in place to avoid the subjects following scent/tracks from the training trials. In Experiment 2 , the general construction was the same, but the horses entered the maze via the $\mathrm{N}$ and S during training trials, with the food located always to the Left (or Right).

group failing to recognise differences in short versus long delays to reinforcement [23].

While these behavioural differences are interesting, supporting physiological data relating to stereotypy as well as adding to our understanding of the physiological workings of learning, they do not increase our understanding of the aetiology or pathogenesis of environmentally induced stereotypy. An insight into these mechanisms may come from additional studies which have demonstrated that dopaminergic agonists not only induce stereotypy [3] but can also cause an accelerated change from response-outcome (R-O) to stimulus-response (S-R) learning [2,20,24]. Work assessing the non-stereotypy behavioural consequences (e.g. effects on learning) of chronic administration of psychostimulants, may in fact be a very insightful method of determining the underlying neurological mechanism of environmentally induced stereotypy [16].

The aim of this study was to examine R-O and S-R learning in horses performing an oral stereotypy (crib-biting), compared to control animals, employing variations of the place/response procedure [21,22]. In the light of the effects of psychostimulant drugs on learning [20] and the neural phenotype found in crib-biting horses [17], we predicted that the crib-biters would display S-R learning in the cross-maze, whereas the non-crib- biting controls would display R-O. We also employed an arena test as a control condition to ensure that crib-biting horses were able to use place learning.

\section{Methods}

Subjects were initially trained in a two-alternative forced choice 'Tolman' crossmaze [26] to locate a food reward in one of two arms. In the second experiment, subjects were trained in a variation of the procedure. This time they were trained, in a discrete-trial format, that food was always located (for example) on the left. This was achieved by changing the point of entry into the maze from trial to trial, in an attempt to nullify the efficacy of using place cues. Finally, an arena field maze test was run with a variety of local and global allocentric cues as a control, in order to ensure that crib-biting horses were able to perform place learning.

\subsection{Subjects}

Ten horses ( $n=3$ crib-biting, $n=3$ non-crib-biting geldings, and $n=2$ crib-biting, $n=2$ non-crib-biting mares) were used for the Experiment 1 . Eight horses ( $n=4 \mathrm{crib}$ biting mares, $n=4$ non-crib-biting mares) were recruited for Experiment 2. Nine horses ( $n=3$ crib-biting mares, $n=2$ crib-biting geldings, and $n=2$ crib-biting, $n=2$ non-crib-biting mares) from two different institutions were recruited for Experiment 3. The ages of all of the horses ranged from 4-25 years. The subjects were selected following an advertisement on an internet-based forum. For each cribbiter a control subjects, that did not display crib-biting, was recruited from the same establishment where possible. Subjects were matched as far as possible for breed and management routine. The latter was achieved by assuring that each control resided with a crib-biter and, in addition, was managed in the same way (i.e., turn-out routine, forage availability, work routine etc.). All animals were fed on a mixture of commercial low-energy feed and ad libitum hay or haylage. All animals were turned out to pasture daily. All aspects of handling of the animals during the three procedures were carried out by the horses' owners (or regular keepers), who were, in each case, blind to the aims and objectives of the experiment.

\subsection{Apparatus and materials}

\subsubsection{Experiments 1 and 2}

The cross-maze $\left(15 \mathrm{~m}^{2}\right)$ was constructed using plastic stakes $(h=60 \mathrm{~cm})$ and nylon tape (see Fig. 1). Each arm of the maze measured $5 \mathrm{~m}$. Four (Experiment 1 ) and two (Experiment 2) identical black plastic buckets were located in each arm of the maze (two in the east arm, two in the west arm for Experiment 1; one in each arm for Experiment 2). The baited bucket contained approximately $5 \mathrm{~g}$ of hifibre commercial concentrate. The mazes were erected within the subjects' own establishments, in a dressage arena or similar. In all cases the mazes were surrounded with a variety of distal cues (e.g., trees, buildings, etc.). A stopwatch was used to measure approach latency to the buckets.

\subsubsection{Apparatus and materials: Experiment 3}

The trials were carried out in rectangular arenas measuring $20 \mathrm{~m} \times 60 \mathrm{~m}$. One arena was located in a disused barn, and the floor was concrete covered with a layer of sand. The second arena was the same dimensions, but located outdoors. Surrounding both arenas were located a large number of distal cues including trees, out-buildings and hedgerows for the outdoor arena and hay-bales, doorways and pillars in the indoor arena. Each of the arenas was divided arbitrarily into quadrants along the horizontal ( $20 \mathrm{~m}$ width-wise) and vertical ( $60 \mathrm{~m}$ length-wise) (see Fig. 2). Two plastic buckets, one yellow and one black, were used in the study and were located either in the NW or SE of the arena. Horses are dichromatic and are able to distinguish between yellow and black objects [15]. The locations of the reinforced $(\mathrm{CS}+)$ and non-reinforced ( $\mathrm{CS}-$ ) buckets were counterbalanced between the yellow and black buckets, and across locations, across subject-pairs.

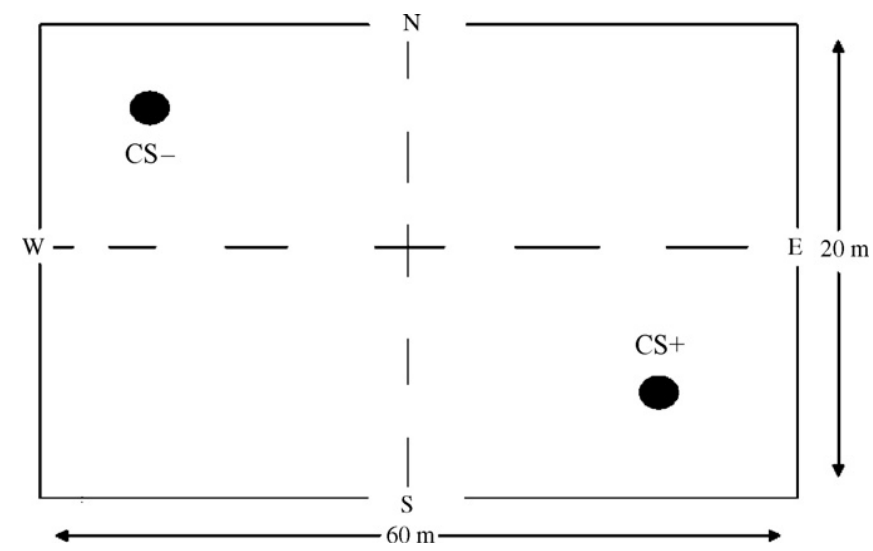

Fig. 2. The dimensions of the arenas used in Experiment 3. The location of the Baited Bucket (CS+) was counterbalanced between the NW and SE across participants. 
2.3. Procedures

\subsubsection{Experiment 1: place/response}

In order to confirm the crib-biting status of the horses, all subjects were given limited access to concentrate food and observed for $5 \mathrm{~min}$. Horses were initially habituated to the maze for approximately $2 \mathrm{~min}$. No food was available during the habituation period. The entry point (i.e., north or south arm) and the baited arm (i.e., east or west) were counterbalanced across participants. Following habituation, subjects were led to an external start point. This position was counterbalanced across subjects (i.e., either adjacent to the east or west arm of the maze). Before being led into the maze in each trial, horses were led around the perimeter of the maze in either a clockwise or anticlockwise direction in a pseudorandom order for each trial in order to avoid generalisation decrement in probe trials. When the horse arrived at the start point (i.e., the entrance to the north or south arm, dependent on counterbalance condition) it was released and allowed freely to traverse the maze. Once the food was located and consumed, the owner entered the maze and led the horse back to the start point. During the initial four training trials, if the horse did not locate the food within a period of 60 -s, the owner entered the maze and guided the horse to the baited bucket. Training trials were carried out in blocks of four with a 2-min break between each block. During the break, the horse stood with the owner by the start point. In total, each horse completed 20 trials in the first session. For each subject, the 20th trial was a probe trial, during which the horse was led to the opposite entry point to the training trials and released in the same manner by the owner. During the probe trial, neither bucket contained food. The food-location latency and proportion of correct trials in each block were collected for each subject. For data analysis, chi-squared tests were carried out with crib-biting status as the independent factor and place or response choice during the probe tria as the dependent variable. In addition, acquisition rate was assessed with location latency (s) in each block as the dependent measure, block as the within-subjects factor and crib-biting status as the between-subjects factor.

\subsubsection{Experiment 2: forced response}

All subjects were screened to assess their crib-biting status (as described in Section 2.3.1). Initially, subjects were led into the maze, directed into the reinforced arm and allowed to consume the food. Following this, the training trials commenced. Unlike Experiment 1, the horses were not allowed to traverse the maze freely to locate the food. There, incorrect choices could have been inadvertently maintained as all trials were reinforced. Owing to the relative simplicity of Experiment 1, this did not prove to be problematic, with all of the subjects quickly learning the location of the food. However, because of the complexity of the current procedure, we implemented a discrete-trial format whereby, following an incorrect response (i.e., the horse choosing the unreinforced arm at the choice point), reinforcement was withheld (the horse was immediately removed from the maze). The order of entry (i.e., north or south) was arranged in random order (e.g., NNSSSNSSNNSSNS etc). The random order was generated through a simple algorithm written in Microsoft Excel. During trials, the arm directly opposite the entry-arm was blocked (i.e., if the horse entered via the south arm, the north arm was blocked). Food was always located either to the left or to the right, and this was counterbalanced across subjects. For example, when a 'turn-left' horse entered via the south arm, food would be located in the west arm, whereas when that horse entered via the north arm, food would be located in the east arm. Horses were trained for 40 trials, split into eight blocks of five. Between each block, horses had a break of $1 \mathrm{~min}$. Training took approximately 45 -min per animal. Owing to the format of the procedure (i.e., discrete-trial), location latency was inappropriate as an operationalisation of learning in this context (as all incorrect trials were non-reinforced). Therefore, the dependent measure was the proportion of errors within each trial block.

\subsubsection{Experiment 3: place-learning control}

Initially, all horses were assessed for crib-biting status (as described in Section 2.3.1). Before commencing training, each subject was led around the perimeter of the arena (see Fig. 2) to habituate them to the set-up. In all subjects, for the first trial, the horse was led to CS+ with a lead-rope and it was ensured that the horse consumed the food. Each horse then took part in nine further training trials. Before each trial, the leader took the horse to one of the four start-points (i.e., north, south, east or west; see Fig. 2). During each training trial the subject was released from its lead-rope, facing the wall, and allowed to explore the arena freely. Following location of $\mathrm{CS}^{+}$, the subject was allowed to consume the food, the lead rope was re-attached, and the horse was led out of the arena. The horse remained outside the arena with the leader for 30-s and was then led back into the arena and to the next start point The order of start points was randomised across trials.

Following training, the subjects took part in three test-trials in extinction. The order of these trials was counterbalanced across subjects, and each was interspersed with a re-training trial. Subjects were released from either the east or west startpoints for all test-trials (see Fig. 2). The proportion of time subjects spent in the correct quadrant of the arena (i.e., the previous location of the baited bucket) was examined for a period of 45-s. For test-trial 1, the horse was introduced to the arena with no buckets $\left(\mathrm{CS}_{0}\right)$ present. For test-trial 2, the buckets were switched (CS-CS+; i.e., in Fig. 2, CS+ would be placed in the SE corner, and CS- in the NW). For test-trial 3 , the arena contained $2 \times \mathrm{CS}+$

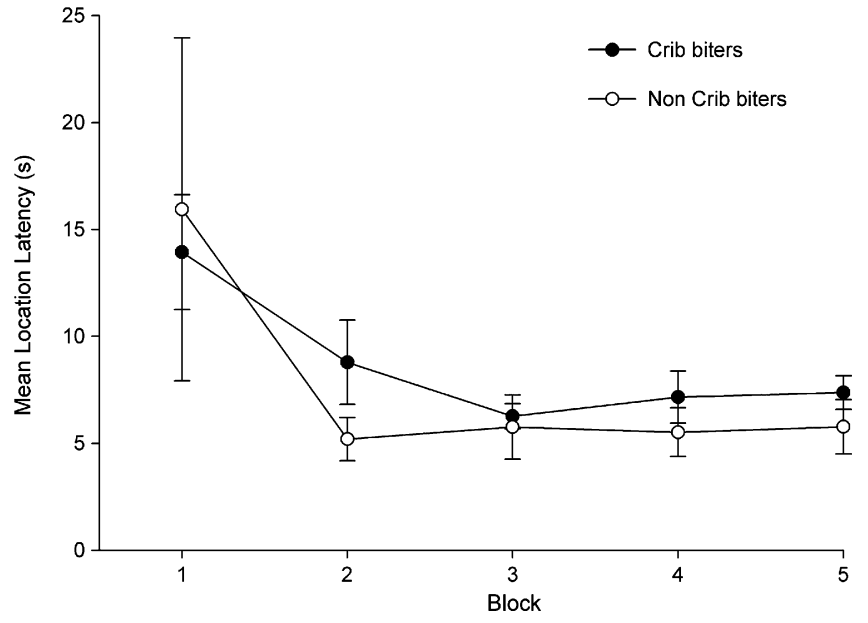

Fig. 3. Mean $( \pm S E M)$ food location latency (s) as a function of trial-block (i.e., four trials) in crib-biting and non-crib-biting horses.

We found large deviations in food-approach latencies between subjects, perhaps as an effect of subjects' differing levels of fitness or motivation. Therefore, for analysis, we operationalised learning as the proportion of deviation (error) from the optimal route (calculated as optimal route length/actual route length). Group (cribbiters and non-crib-biters) was the independent factor. During the three probe trials, place learning was operationalised as time spent in the previously reinforced quadrant of the arena (i.e., previous location of $\mathrm{CS}^{+}$) again with group as the independent factor.

\section{Results}

\subsection{Experiment 1: place-response}

Fig. 3 displays the acquisition rates for the two groups. Acquisition data were $\log$ (base 10) transformed prior to analysis to normalise the distribution. A mixed design $2 \times 5$ Analysis of Variance (ANOVA), with crib-biting status as the between-subjects factor and block as the within-subjects factor, revealed a significant main effect for block, $F(4,32)=5.81, P<0.05$, but not for group, nor group $\times$ block interaction $(F s<1)$. This suggests that both groups learned to locate the food at similar rates, with the location latency typically reducing to stabilise after the first block of learning trials.

The results from the probe trial are illustrated in Fig. 4. A chisquared test showed that there was a significant difference between the groups, with more of the crib-biters displaying response learning $(n=3)$ than non-crib-biters $(n=1), \chi^{2}(1)=4.29, P<0.05$.

\subsection{Experiment 2: forced response}

Acquisition graphs from Experiment 2 are displayed in Fig. 5. All proportion data were arcsine-root transformed prior to analysis to normalise the distribution. The data were analysed using a mixed-design, $2 \times 8$ ANOVA, with crib-biting status as the betweensubjects factor and trial block as the within-subjects factor. We found a significant main effect for trial block, $F(7,42)=5.79$, $P<0.01$ and for status, $F(1,6)=10.12, P<0.05$, and a significant trial block $\times$ status interaction, $F(7,42)=2.26, P<0.02$. Simple main effects analyses [13] revealed that the crib-biters made significantly fewer errors than the non-crib-biters in the third, seventh and eighth trial blocks $\left(P_{\mathrm{s}}<0.05\right)$.

\subsection{Experiment 3: place learning control}

Prior to analysis, error-proportion data were arcsine-route transformed to normalise the distribution. 

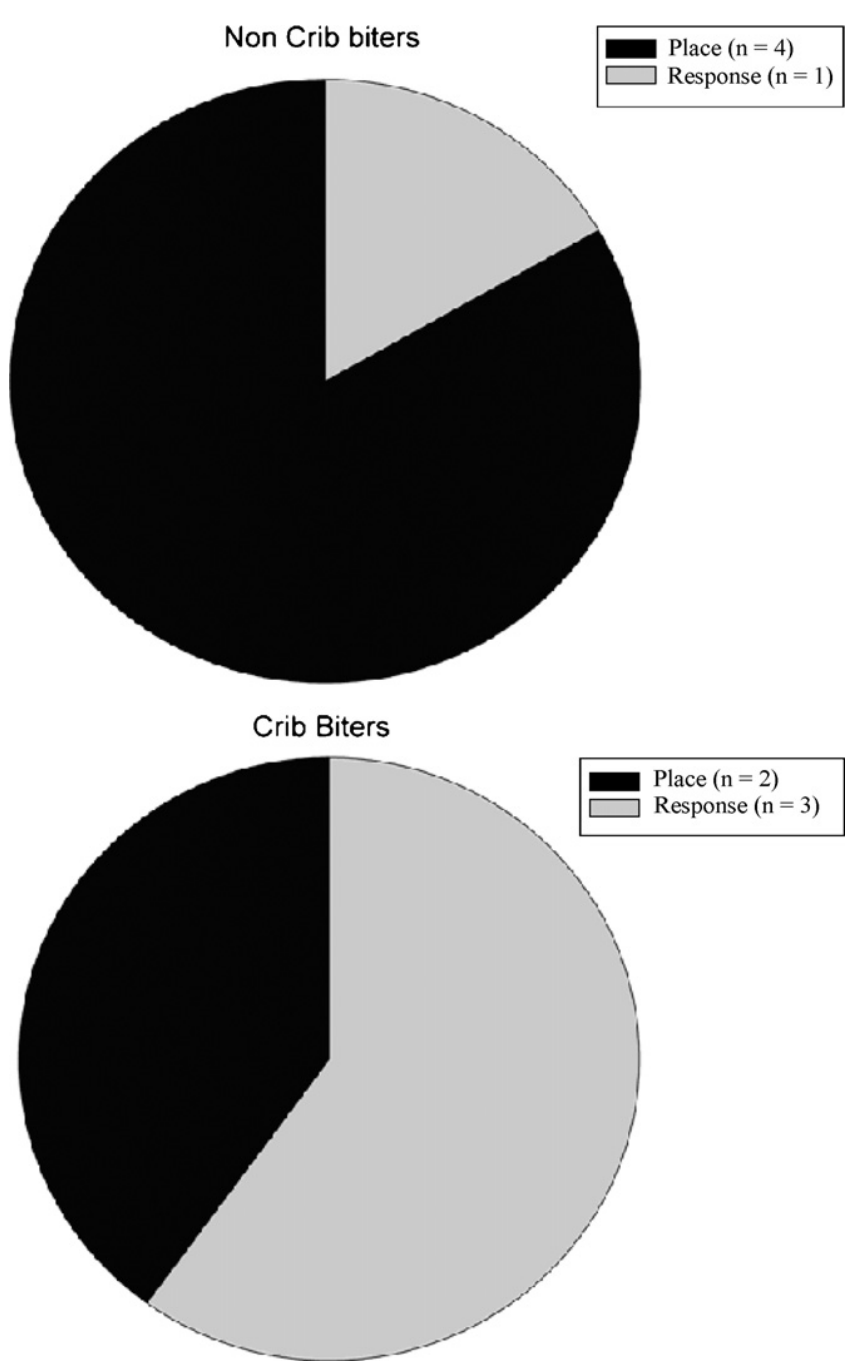

Fig. 4. Number of horses in each group that showed place or response learning in the probe trial.

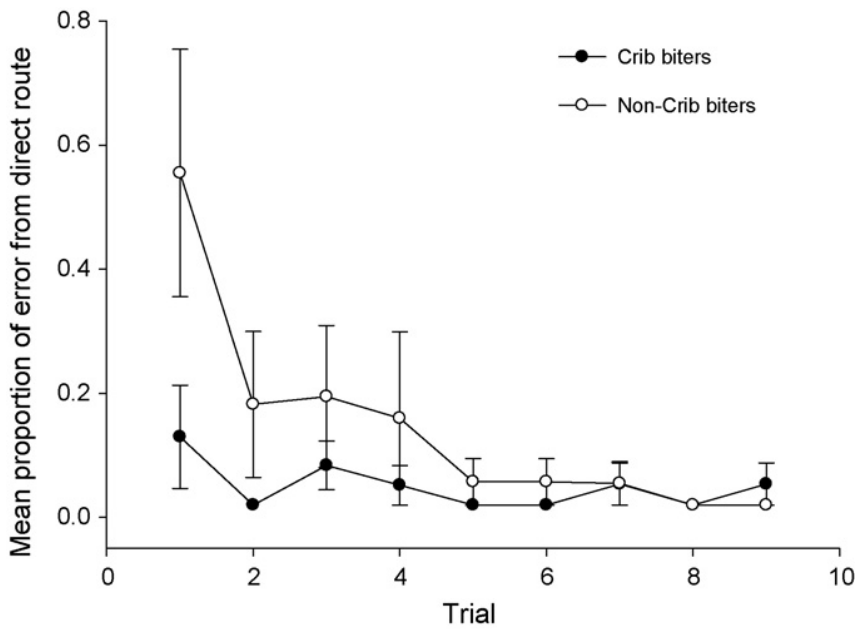

Fig. 6. Mean $( \pm S E M)$ proportion of error from optimal route of crib-biters and noncrib-biters in open field maze.

Training data are displayed in Fig. 6. The proportion of error from the optimal route for both groups across the nine training trials was examined using a mixed design 2 (group) $\times 9$ (trial) ANOVA. There was a significant main effect for trial, $F(8,56)=5.14, P<0.05$, and a significant trial $\times$ group interaction, $F(8,56)=2.70, P<0.05$. There was no main effect found for group $F(1,7)=4.33, P>0.05$. Post hoc analyses, corrected for multiple tests, revealed that subjects made a lower proportion of error in trial 1 as compared to trials 2-9 (all Ps $<0.05$ ).

During the three probe trials, use of global place cues was operationalised as time spent in the previously reinforced quadrant of the arena (i.e., previous location of CS+). Fig. 7 illustrates the proportion of time spent in each quadrant during the probe trials for each group. A mixed design $2 \times 3$ ANOVA, with crib-biting status as the between-subjects factor, probe trials as the within-subjects factor and proportion of time spent in the correct quadrant as the dependent measure, revealed no significant main effects for probe trial or for group, nor was there an interaction (all $F s<1$ ). In addition, we employed an additional $2 \times 3$ ANOVA to examine the latency to approach the area of the arena where CS+ had been previously located between the groups (see Fig. 8). This revealed no significant main effects for group or trial, nor was there a group $\times$ trial interaction (all Fs $<1$ ).

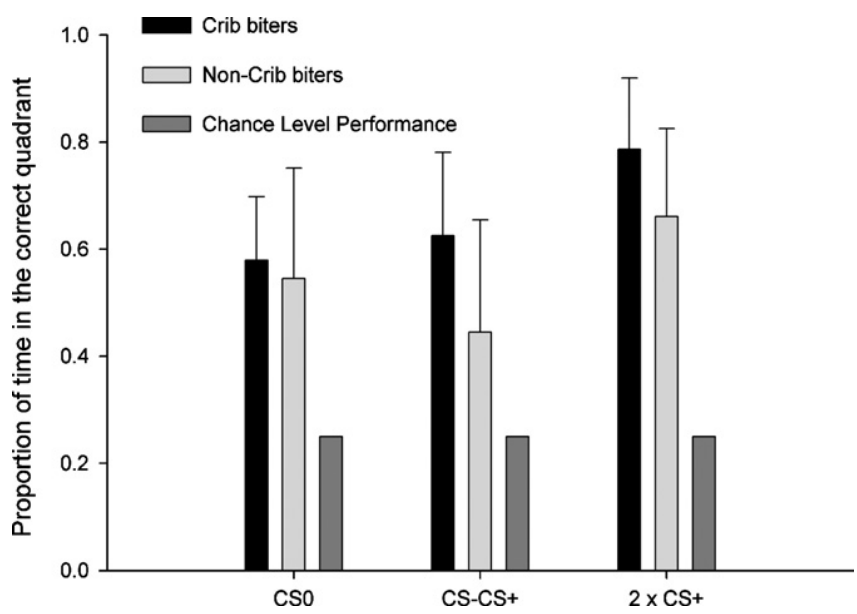

Fig. 7. Mean $( \pm S E M)$ proportion of time spent in the quadrant where food had been previously located (correct quadrant) during three probe trials. Chance Level Performance was set at 0.25 .
Fig. 5. Mean $( \pm$ SEM) proportion of correct trials in each trial block ( 5 trials) for the crib-biters and non-crib-biters.

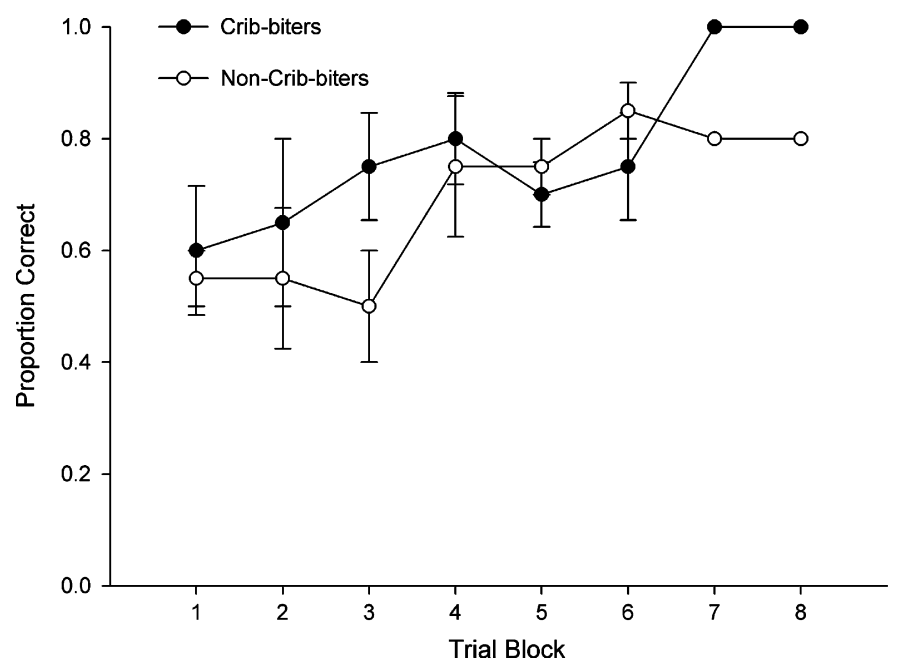




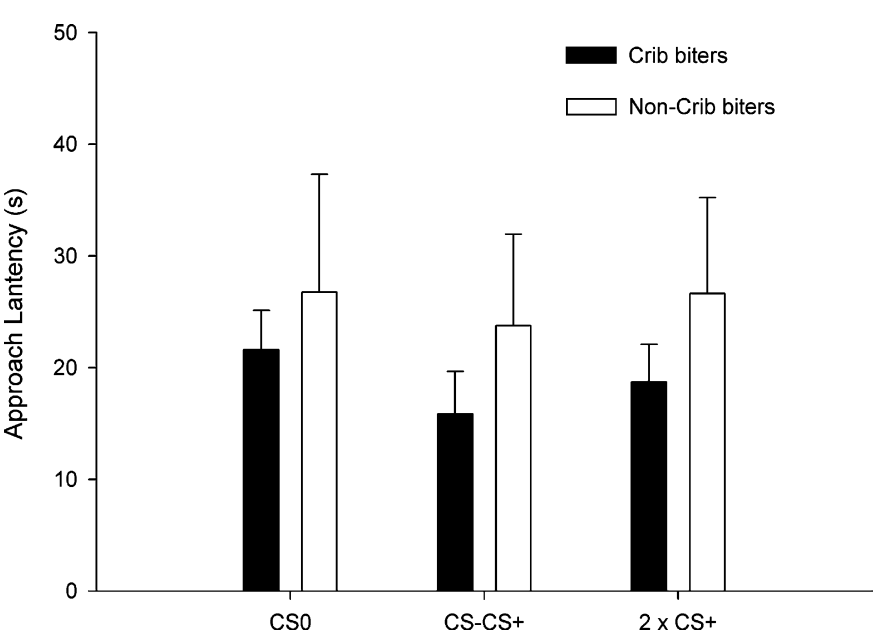

Fig. 8. Mean ( \pm SEM) approach latencies (s) during probe trials.

One-sample t-tests, with the test value set at 0.25 (see Fig. 7), revealed that the crib-biters spent significantly more time than would be predicted by chance in the correct quadrant for $\mathrm{CS}_{0}, t$ $(4)=2.77, P<0.05$, and for $2 \times C S+, t(4)=4.03, P<0.05$ conditions. They also spent marginally more time than would be predicted by chance in the correct quadrant in the CS-CS+ condition, but this difference fell short of statistical significance, $t(4)=2.39, P=.07$. For the non-crib-biters, there was no significant difference from chance in the $\mathrm{CS}_{0}, t(3)=1.43, P>0.05$, and CS-CS,$+ t<1$, conditions, nor for the $2 \times C S+$ condition, $t(3)=2.50, P>0.05$.

\section{Discussion}

The aim of the present studies was to examine learning and behavioural processes associated with the equine oral stereotypy phenotype. Given the previously identified neural characteristics of this group [17], we predicted that crib-biting horses would adopt a response strategy early in the learning process. Using two modified versions of the Tolman cross-maze, we were able to demonstrate this successfully. We also found that crib-biting horses are able to use place-cues in a standard field-maze, showing marginally faster place learning than the controls. These findings are interesting in the light of previous research which has found similar learning differences to be correlated with drug-induced stereotypy.

The results of Experiments 1 and 2 were the first to reveal that horses displaying an environmentally induced stereotypy show similar learning patterns to dopamine agonist-exposed animals. In Experiment 1, for example, only one of the non-crib-biters adopted a response strategy, whereas three of the crib-biters did so. However, these findings are somewhat inconclusive, as only just over half of the crib-biters displayed S-R-like learning. In Experiment 2, where the animals were forced to adopt a response strategy in order to learn the task effectively, the crib-biters were found to learn the task more effectively. Taken together, and notwithstanding the low sample size, it appears that crib-biters were preferentially using S-R learning, whereas the non-crib-biters, R-O.

The results of Experiment 3 demonstrated that crib-biters showed marginally enhanced place learning. This demonstrated that the results of Experiments 1 and 2 were not because the crib-biters failed to learn about 'place'. The reason the crib-biters spent more time in the correct quadrant during probe trials and learnt the correct location of the food at a faster rate may be that they showed a decrease in exploratory behaviour. This may be equated to the behavioural effects of dopamine agonists, where the response repertoire narrows with increasing doses [14]. More research on place learning in animals displaying environmentally induced stereotypies may elucidate this in the future.

Crib-biting horses have significantly higher D1 and D2 receptorsubtypes in the ventral striatum and significantly lower D1 receptor subtypes in the caudate [17]. Therefore, the apparent enhanced S-R learning observed here might be explained by this structural alteration in the striatum. The three systems within the striatum are organised hierarchically [29]. The limbic network, which incorporates the ventral striatum (nucleus accumbens), with excitatory input from the pre-frontal cortex; the associative network, which incorporates the caudate, with excitatory input from the pre-frontal and parietal cortices; finally, the sensorimotor network, which incorporates the putamen, with excitatory input from the sensorimotor cortex. During habit formation, there is a shift through the hierarchy, from initial Pavlovian processes, through RO judgements, finally to automated, S-R habitual responses $[28,29]$. Although our results do not clearly demonstrate that the horses' learning shifts from R-O to S-R during the course of the trials, they may indicate that, in the crib-biting phenotype, $\mathrm{R}-\mathrm{O}$ learning is superseded by S-R learning.

It has recently been established that during the shift between $\mathrm{R}-\mathrm{O}$ and $\mathrm{S}-\mathrm{R}$ learning there is a change in balance between matrix and striosome cell activation in the striatum, and this alteration in balance correlates highly with drug-induced stereotypy (e.g., see $[2-6,10,25])$. This behavioural and physiological alteration in functioning is enhanced by chronic amphetamine exposure [2], and thus the shift between R-O and S-R learning, and the progressive imbalance of striosome and matrix cells, are mediated through dopaminergic pathways. The behavioural data presented in this study may suggest that horses which display and oral stereotypy also have a similar imbalance of striosome and matrix cells. Clearly, the differences in $\mathrm{R}-\mathrm{O}$ to $\mathrm{S}-\mathrm{R}$ learning may be no more than an artifact of the crib biting phenotype [17]. However, chronic coactivation of dopamine D1 and D2 receptors using agonist drugs results in striosome predominance and locomotor stereotypy in both drug free and cocaine pre-treated rats [6]. This may suggest an aetiological pathway for the environmentally induced stereotypy phenotype, with facilitated dopamine transmission, as a result of an interaction of genotype and stress-exposure, leading to striosome predominance in the striatum. This may warrant further physiological research.

Finally, we appreciate that the external validity of this is limited by the small sample size and concede that this limits the generalisability of the findings. However, these findings represent an important first step towards a more clear understanding of the behavioural processes associated with endogenous basal ganglia dysfunction and may have important implications for further research into the development of conceptual aetiological models of spontaneous stereotypy.

\section{Acknowledgements}

This research was funded by an ESRC Ph.D. studentship (MP). The authors would like to thank all of the owners and yards involved in the study, particularly staff at Sparshlolt College Equine Centre, for the use of their horses and facilities. Thanks also to Dr Emily O'Connor, Professor Roger Parker and two anonymous reviewers for helpful comments on earlier drafts of this paper.

\section{References}

[1] Cabib S, Giardino L, Calza L, Zanni M, Mele A, Puglisi-Allegra S. Stress promotes major changes in dopamine receptor densities within the mesoaccumbens and nigrostriatal systems. Neuroscience 1998:84:193-200.

[2] Canales JJ. Stimulant-induced adaptations in neostriatal matrix and striosome systems: transiting from instrumental responding to habitual behavior in drug addiction. Neurobiol Learn Mem 2005;83:93-103. 
[3] Canales JJ, Capper-Loup C, Hu D, Choe ES, Upadhyay U, Graybiel AM. Shifts in striatal responsivity evoked by chronic stimulation of dopamine and glutamate systems. Brain 2002;125:2353-63.

[4] Canales JJ, Graybiel AM. A measure of striatal function predicts motor stereotypy. Nat Neurosci 2000;3:377-83.

[5] Canales JJ, Iversen SD. Psychomotor-activating effects mediated by dopamine $\mathrm{D}(2)$ and $\mathrm{D}(3)$ receptors in the nucleus accumbens. Pharmacol Biochem Behav 2000;67:161-8.

[6] Capper-Loup C, Canales JJ, Kabada N, Graybiel AM. Concurrent activation of dopamine D1 and D2 receptors is required to evoke neural and behavioural phenotypes of cocaine sensitization. J Neurosci 2002;22:6218-27.

[7] Cooper SJ, Dourish CT. Neurobiology of Stereotyped Behaviour. Oxford: Oxford Science Publications; 1990, 297 pp.

[8] Di Chiara G. Dopamine, motivation and reward. In: Dunnett SB, editor. Handbook of Chemical Neuroanatomy, 21. New York: Elsevier; 2005. p. 303-94.

[9] Garner JP, Mason GJ, Smith R. Stereotypic route-tracing in experimentally caged songbirds correlates with general behavioural disinhibition. Anim Behav 2003;66:711-27.

[10] Graybiel AM. Habits, rituals, and the evaluative brain. Ann Rev Neurosci 2008;31:359-87.

[11] Hemmings A, McBride SD, Hale CE. Perseverative responding and the aetiology of equine oral stereotypy. App Anim Behav Sci 2007;104:143-50.

[12] Houpt KA, Mcdonnell SM. Equine Stereotypies. Compendium Contin Educ Practicing Veterinarian 1993;15:1265-72.

[13] Keppel G. Design and Analysis: A Researcher's Handbook. Englewood Cliffs, NJ: Prentice-Hall; 1973, 624 pp.

[14] Lyon M, Robbins T. The action of central nervous system drugs: a general theory concerning amphetamine effects. Curr Dev Psychopharmacol 1975:80-163.

[15] Macuda T, Timney B. Luminance and chromatic discrimination in the horse (Equus caballus). Behav Process 1999;44:301-7.

[16] Mason GJ, Rushen J. Stereotypic Animal Behaviour: Fundamentals and Applications to Welfare, 2. Oxford: CABI; 2006, 384 pp.

[17] McBride SD, Hemmings A. Altered mesoaccumbens and nigro-striatal dopamine physiology is associated with stereotypy development in a nonrodent species. Behav Brain Res 2005;159:113-8.
[18] McGreevy PD, Cripps PJ, French NP, Green LE, Nicol CJ. Management factors associated with stereotypic and redirected behavior in the thoroughbred horse. Eq Vet J 1995;27:86-91.

[19] McGreevy PD, Richardson JD, Nicol CJ, Lane JG. Radiographic and endoscopic study of horses performing an oral based stereotypy. Eq Vet J 1995;27:92-5.

[20] Nelson A, Killcross S. Amphetamine exposure enhances habit formation. J Neurosci 2006;26:3805-12.

[21] Packard MG, Knowlton BJ. Learning and memory functions of the basal ganglia. Ann Rev Neurosci 2002;25:563-93.

[22] Packard MG, McGaugh JL. Inactivation of hippocampus or caudate nucleus with lidocaine differentially affects expression of place and response learning. Neurobiol Learn Mem 1996;65:65-72.

[23] Parker M, Redhead ES, Goodwin D, McBride SD. Impaired instrumental choice in crib biting horses (Equus caballus). Behav Brain Res 2008;191:137-40.

[24] Robbins TW, Everitt BJ. A role for mesencephalic dopamine in activation: commentary on Berridge (2006). Psychopharmacology 2007;191:433-7.

[25] Saka E, Goodrich C, Harlan P, Madras BK, Graybiel AM. Repetitive behaviours in monkeys are linked to specific striatal activation patterns. J Neurosci 2004;24:7557-65.

[26] Tolman EC. The determinants of behavior at a choice point. Psych Rev $1938 ; 45: 1-41$.

[27] Vickery SS, Mason G. Stereotypy and perseverative responding in caged bears: further data and analyses. App Anim Behav Sci 2005;91:247-60.

[28] Yin HH, Knowlton BJ. Contributions of striatal subregions to place and response learning. Learn Mem 2004;11:459-63.

[29] Yin HH, Knowlton BJ. The role of the basal ganglia in habit formation. Nat Rev Neurosci 2006;7:464-76.

[30] Yin HH, Knowlton BJ, Balleine BW. Lesions of dorsolateral striatum preserve outcome expectancy but disrupt habit formation in instrumental learning. Eur J Neurosci 2004;19:181-9.

[31] Yin HH, Knowlton BJ, Balleine BW. Inactivation of dorsolateral striatum enhances sensitivity to changes in the action-outcome contingency in instrumental conditioning. Behav Brain Res 2006;166:189-96.

[32] Yin HH, Ostlund SB, Knowlton BJ, Balleine BW. The role of the dorsomedial striatum in instrumental conditioning. Eur J Neurosci 2005;22:513-23. 